Topol ogy Opt i mizat i on of Opt i cal Wavegui de Devi ces Based on Beam Propagat i on Met hod With Sensi ti vity Anal ysi s

\begin{tabular}{|l|l|}
\hline 著者 & $\begin{array}{l}\text { I GUCH Aki to, TSUJ I Yasuhi de, YASUl Takashi , } \\
\text { H RAYAMA Koi chi }\end{array}$ \\
\hline $\begin{array}{l}\text { j our nal or } \\
\text { publ i cat i on t i t l e }\end{array}$ & Journal of Li ght wave Technol ogy \\
\hline vol une & 34 \\
\hline number & 18 \\
\hline page range & $4214-4220$ \\
\hline year & $2016-09-15$ \\
\hline URL & ht t p: //hdl . handl e. net /10258/00009485 \\
\hline
\end{tabular}




\title{
Topology Optimization of Optical Waveguide Devices Based on Beam Propagation Method With Sensitivity Analysis
}

\author{
Akito IGUCHI, Student member, IEEE, Yasuhide TSUJI, Member, IEEE, Member, OSA, Takashi YASUI, Member, \\ IEEE and Koichi HIRAYAMA, Senior Member, IEEE
}

\begin{abstract}
We present a gradient-based topology optimization method of optical waveguide devices using the beam propagation method (BPM). Efficient topology optimization of an optical waveguide is available by using our approach because it utilizes the adjoint variable method (AVM) for sensitivity analysis, and the BPM for numerical propagation analysis. In this paper, a way of evaluating sensitivities for a design of longitudinally varying waveguides is newly described in the case that a density method and finite difference BPM (FD-BPM) are employed. In addition, the validity and availability of our proposed method are verified by optimizing an S-bend and a Y-branch waveguides.
\end{abstract}

Index Terms-Topology optimization, Adjoint variable method, Beam propagation method, Optical waveguide device.

\section{INTRODUCTION}

In resent years, high speed and large capacity communication systems are required. High performance optical waveguide devices are in demand to realize these systems. Recently, the optimal design of optical waveguide devices based on the topology optimization method has attracted lots of attention. Compared with size or shape optimization, the advantage of the topology optimization method is that it has a great flexibility. Therefore, there is a potential for designing the unconventional and high performance devices by using the topology optimization.

Topology optimization method has already been applied to design optical waveguide devices such as photonic crystal waveguide devices [1]- [6]. We also have reported the application to optical waveguides and confirmed the validity of topology optimization [7]- [11]. Although the finite element method (FEM) or finite difference time domain (FDTD) method are used as a propagation analysis method in these reports, topology optimization does not restrict a numerical calculation method to the FEM or FDTD. Thus one can select a suitable wave propagation analysis method depending on a design problem in topology optimization.

Beam propagation method (BPM) is a quite useful technique in the case that backward wave reflections are negligible and refractive index varies slowly along the propagation direction. The wavefront matching (WFM) method [12], [13], which

A. Iguchi and Y. Tsuji are with the Division of Information and Electronic Engineering, Muroran Institute of Technology, Muroran, 050-8585 Japan (email: 15043003@mmm.muroran-it.ac.jp; y-tsuji@mmm.muroran-it.ac.jp). T. Yasui and K. Hirayama are with the Division of Electrical and Electronic Engineering, Kitami Institute of Technology, Kitami 090-8507, Japan (e-mail: yasui@mail.kitami-it.ac.jp; hirakc@mail.kitami-it.ac.jp). utilizes a principle of the BPM, is proposed as an optimal design method of weakly guiding optical waveguides. An optimum refractive index distribution can be synthesized automatically using the WFM. Several applications using the WFM method has been reported, for instance, a Y-branch waveguide [14], a crossing waveguide [15], and an arrayedwaveguide grating [16] for integrated planar lightwave circuits. The optimal design technique in [12] has a disadvantage in that a complicated refractive index distribution which is called "mosaic-like pattern" is appeared. This drawback is to some extent overcome by using "Solid pattern" approach proposed in [13]. However, WFM is not based on an objective function minimization problem, and the arbitrariness of objective property has not been discussed yet.

Topology optimal design problem is able to be efficiently solved by using a gradient method like a steepest descent if sensitivities for the design parameters are easily evaluated. Sensitivities of enormous design parameters have to be calculated in gradient-based topology optimization. In the topology optimization process, it is not practical to approximate a lot of sensitivities by finite difference scheme, therefore, an adjoint variable method (AVM) can be utilized for analyzing the sensitivities efficiently. A size optimization of optical waveguides using the AVM and BPM has already been proposed [17], [18]. However, topology optimization based on these approaches has not yet been discussed.

In this paper, we describe a topology optimal design method using the AVM and finite difference BPM (FD-BPM). A general way of evaluating sensitivities with a central AVM (CAVM) and the BPM has been described in [17], [18] for the optimization of the size or refractive index. In our approach, a density method, which is widely used in the topology optimization, is used as a representation of refractive index distribution and we describe a way to evaluate sensitivities for a longitudinally varying waveguide without using CAVM in the case that the density method is employed. By using our approach, an optical waveguide device can be designed using the topology optimization even if the device is too long to be analyzed with reasonable computer resources by the FEM or FDTD. We confirm the validity of our proposed topology optimal approach by applying it to a design of an S-bend and a Y-branch waveguides.

In Section II, we firstly review the formulation of FDBPM, then a way of a representation of the refractive index distribution is described. Moreover, sensitivity analysis method 


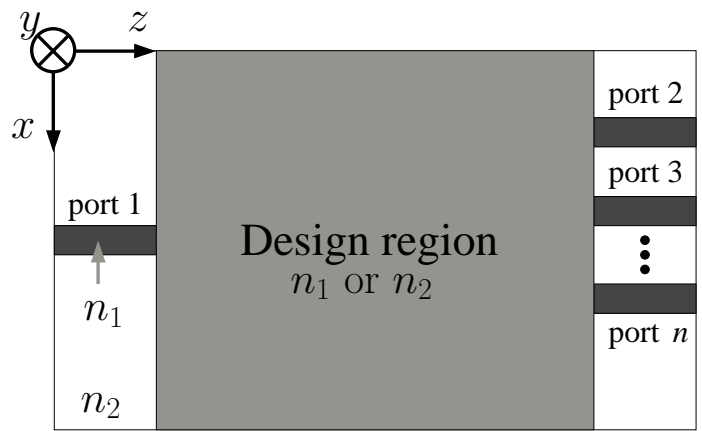

Fig. 1. Topology optimal design model of a 2-D optical waveguide.

in the case of using the FD-BPM and the density method is described in Section III. After that, the design examples of the application of our approach are shown in Section IV. Finally, we state the conclusion in Section V.

\section{TOPOLOGY OPTIMIZATION}

\section{A. Formulation of the FD-BPM}

Now we consider a two dimensional planar optical waveguide, as shown in Fig. 1. In isotropic, linear and non-dispersive dielectric media, 2-D scalar Helmholtz equation for TE and TM polarized waves is given as follows:

$$
\begin{array}{cc}
\frac{\partial}{\partial x}\left(p \frac{\partial \Phi}{\partial x}\right)+\frac{\partial}{\partial z}\left(p \frac{\partial \Phi}{\partial z}\right)+ & k_{0}^{2} q \Phi=0, \\
p=1, q=n^{2}, \Phi=E_{y} & \text { for TE wave, } \\
p=1 / n^{2}, q=1, \Phi=H_{y} & \text { for TM wave, }
\end{array}
$$

where $k_{0}$ is a free-space wavenumber, $n(x, z)$ is a refractive index distribution. By assuming $\partial n / \partial z \approx 0$ and $\Phi(x, z)=$ $\phi(x, z) \exp \left(-j k_{0} n_{0} z\right)$, the following basic BPM equation is obtained,

$$
-\frac{\partial^{2} \phi}{\partial z^{2}}+j 2 k_{0} n_{0} \frac{\partial \phi}{\partial z}=\left(D_{x x}+\nu\right) \phi
$$

with

$$
\begin{aligned}
D_{x x} & =\frac{1}{p} \frac{\partial}{\partial x}\left(p \frac{\partial}{\partial x}\right), \\
\nu & =k_{0}^{2}\left(n^{2}-n_{0}^{2}\right),
\end{aligned}
$$

where $n_{0}$ is a reference refractive index. In the paraxial BPM equation, $\partial^{2} \phi / \partial z^{2}$ is omitted. Then, the following sequential update equation of the BPM can be obtained by using implicit FD scheme for Eq. (2) [19],

$$
[A]_{i}\{\phi\}_{i+1}=[B]_{i}\{\phi\}_{i},
$$

where $[A]_{i}$ and $[B]_{i}$ are the matrices obtained by spatial discretization, $i\left(=0,1, \cdots, N_{z}-1\right)$ is the step number of the $z$-direction.

In this paper, we employ the paraxial approximation on the basic BPM equation. In addition, the transverse direction is discretized by using a FD scheme proposed by Stern [20]. The transparent boundary condition [21] is employed as an absorbing boundary condition.

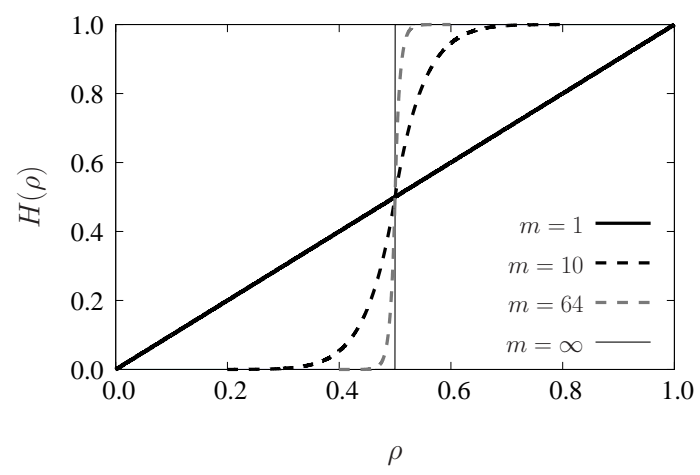

Fig. 2. Examples of Heaviside filter.

\section{B. Representation of refractive index distribution}

In the topology optimization, the representation of refractive index distribution is one of the most important issues. Here, we employ the density method to describe the refractive index in design region. The refractive index distribution is represented by the normalized density distribution $\rho(x, z)$ [1]. A drawback of conventional density method is that a gray region, in which $0<\rho<1$, appears. In order to suppress the gray regions, we express the refractive index distribution by using a Heaviside filter $H(\rho)$ as follows:

$$
\begin{gathered}
n^{2}(x, z)=n_{2}^{2}+\left(n_{1}^{2}-n_{2}^{2}\right) H(\rho), \\
H(\rho)=\left\{\begin{array}{ll}
0.5(2 \rho)^{m} & (0 \leq \rho<0.5) \\
1-0.5(2-2 \rho)^{m} & (0.5 \leq \rho \leq 1)
\end{array},\right.
\end{gathered}
$$

where $m$ is a penalty parameter, $n_{1}$ and $n_{2}$ are the refractive indices of the core and cladding region, respectively. In the practical optimization, the density distribution is discretized on the finite difference grids. As shown in Fig. 2, $H(\rho)$ is a step-like function approximately if $m$ is a large value. Thus, a binarized refractive index distribution is obtained by $m \rightarrow \infty$. However, $n(x, z)$ has to be differentiated by the density parameter in the sensitivity analysis, thus the topology is not freely modified in the case that the value of $m$ is raised excessively. Therefore, in this paper, $m$ is taken to be a lower value in the initial phase of the optimization. Then, the value of $m$ is raised as iterating the optimization, finally, $m$ is set to infinity.

\section{SEnSitivity ANALYSIS}

Now we describe the sensitivity analysis method in the case of using the 2-D BPM and the density method. We assume the design parameters are allocated for each discrete point in the design region. A sensitivity of an objective function $C$ is defined by $\partial C / \partial \rho_{i, j}$ where $\rho_{i, j}$ is a density parameter, $j(=$ $\left.0,1,2, \cdots, N_{x}-1\right)$ is an index number along the $x$-direction. Properties of optical waveguides are usually represented by an S-parameter, i.e. $S_{n 1}$, where $n$ is the output port number. Thus, we consider that $C$ depends on the normalized power $\left|S_{n 1}\right|^{2}$ in the port $n$. The sensitivity of $\left|S_{n 1}\right|^{2}$ is expressed by

$$
\frac{\partial\left|S_{n 1}\right|^{2}}{\partial \rho_{i, j}}=\operatorname{Re}\left\{2 S_{n 1}^{*} \frac{\partial S_{n 1}}{\partial \rho_{i, j}}\right\}
$$


where * means complex conjugate. $S_{n 1}$ can be represented by the following overlap integral,

$$
S_{n 1}=\int \psi_{n}^{*} \phi_{N_{z}} d x
$$

where $\psi_{n}$ is an eigenmode field in the port $n$. In the case that FD scheme is employed, by using the rectangle rule, Eq. (6) can be rewritten as follows:

$$
S_{n 1}=\Delta x\left\{\psi_{n}\right\}^{\dagger}\{\phi\}_{N_{z}},
$$

where ${ }^{\dagger}$ means Hermitian transpose, $\Delta x$ is a step width along the $x$-direction. By substituting Eq. (3) into Eq. (7), we can obtain

$$
\begin{gathered}
S_{n 1}=\Delta x\left\{\psi_{n}\right\}^{\dagger}\left([A]^{-1}[B]\right)_{N_{z}-1}\left([A]^{-1}[B]\right)_{N_{z}-2} \cdots \\
\cdots\left([A]^{-1}[B]\right)_{i} \cdots\left([A]^{-1}[B]\right)_{1}\left([A]^{-1}[B]\right)_{0}\{\phi\}_{0} .
\end{gathered}
$$

By taking the derivative of $S_{n 1}$ with respect to a density parameter $\rho_{i, j}$, we get

$$
\begin{aligned}
\frac{\partial S_{n 1}}{\partial \rho_{i, j}}=\Delta x\left\{\psi_{n}\right\}_{i+1}^{\dagger}( & -[A]_{i}^{-1} \frac{\partial[A]_{i}}{\partial \rho_{i, j}}\{\phi\}_{i+1} \\
& \left.+[A]_{i}^{-1} \frac{\partial[B]_{i}}{\partial \rho_{i, j}}\{\phi\}_{i}\right),
\end{aligned}
$$

where

$$
\begin{array}{r}
\left\{\psi_{n}\right\}_{i+1}^{\dagger}=\left\{\psi_{n}\right\}^{\dagger}\left([A]^{-1}[B]\right)_{N_{z}-1}\left([A]^{-1}[B]\right)_{N_{z}-2} \\
\cdots\left([A]^{-1}[B]\right)_{i+1} .
\end{array}
$$

Here, $\left\{\psi_{n}\right\}_{i+1}$ is a backward propagating field of $\left\{\psi_{n}\right\}$ which comes from the output port $n$. Figure 3 shows the sensitivity analysis using the BPM. $\{\phi\}_{i},\{\phi\}_{i+1}$, and $\left\{\psi_{n}\right\}_{i+1}$ in Eq. (9) are obtained by the forward and backward beam propagations. The refractive index distribution is represented by Eq. (4), thus $\partial[A]_{i} / \partial \rho_{i, j}$ and $\partial[B]_{i} / \partial \rho_{i, j}$ can be calculated analytically without using CAVM. After calculating forward and backward wave propagation fields, the sensitivities for all design parameters can be evaluated by simple matrix-vector products. Therefore, efficient topology optimization can be achieved by using this approach.

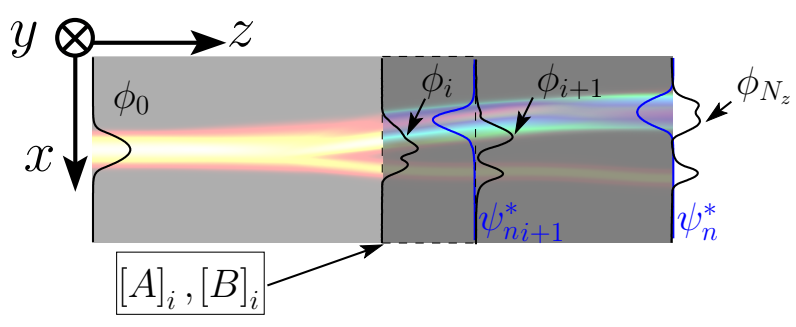

Fig. 3. Sensitivity analysis using the BPM.

\section{DESIGN EXAMPLES}

In this section, in order to demonstrate the validity of our approach, we consider two design examples of weakly guiding optical waveguides. In these examples, a steepest descent

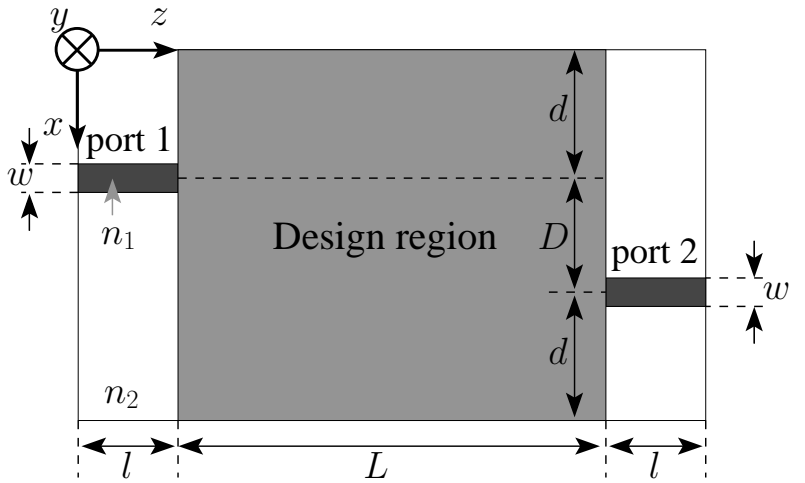

Fig. 4. A design model of a 2-D S-bend waveguide.

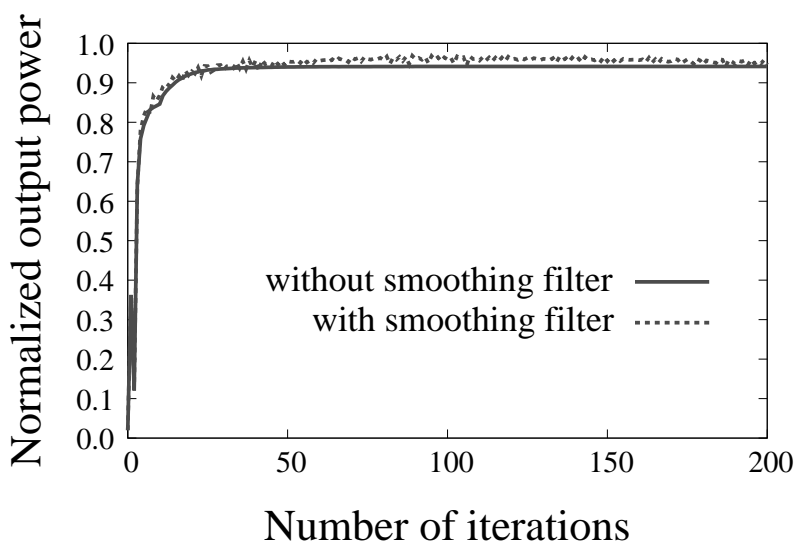

Fig. 5. The normalized output power of the S-bend waveguide in port 2 as a function of the number of iterations.

method is employed to minimize an objective function. The design parameters are updated by following equation:

$$
\rho_{i, j}^{M+1}=\rho_{i, j}^{M}-\frac{1}{K} \frac{\partial C}{\partial \rho_{i, j}},
$$

where $M$ is an iteration number, $K$ is a constant which is taken to be as follows:

$$
K=\max _{i, j \in \Omega}\left|\frac{\partial C}{\partial \rho_{i, j}}\right|,
$$

where $\Omega$ represents a set of discrete points in the design region. After updating the density parameters, $\rho_{i, j}$ is reset to $0+\delta$ or $1-\delta$ in the case of $\rho_{i, j} \leq \delta$ or $1-\delta \leq \rho_{i, j}$, respectively, where $\delta$ is an arbitrarily small value and is introduced for avoiding the sensitivity to be zero. In this paper, we select $\delta$ to be $10^{-8}$. The above constants, $K, \delta$, of course, have to be changed properly depending on a design problem.

\section{A. S-bend waveguide}

Now we design a 2-D S-bend waveguide whose design model is shown in Fig. 4. We assume a fundamental TE mode ( $\mathrm{TE}_{0}$ mode) at the wavelength of $1.55 \mu \mathrm{m}$ is launched into port 1 . The core and cladding refractive indices are $n_{1}=1.45$ and $n_{2}=1.445$, respectively. The configuration parameters of the design model are as follows: $w=5 \mu \mathrm{m}, L=800 \mu \mathrm{m}$, 


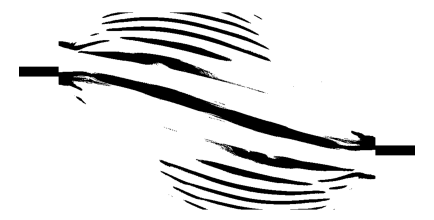

(a) Optimized refractive index distribution

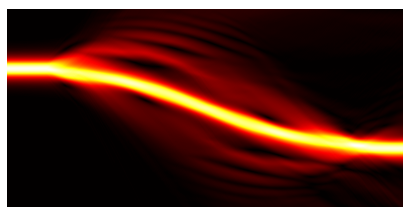

(b) Field pattern $\left(\left|E_{y}\right|\right)$

Fig. 6. The refractive index distribution and the field pattern of the optimized S-bend waveguide.

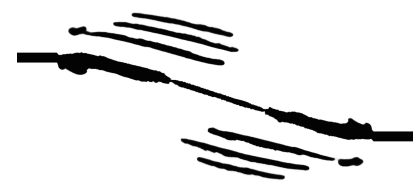

(a) Optimized refractive index distribution

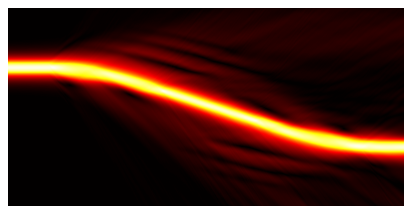

(b) Field pattern $\left(\left|E_{y}\right|\right)$

Fig. 7. The refractive index distribution and the field pattern of the optimized S-bend waveguide using a smoothing filter.

$d=30 \mu \mathrm{m}, l=100 \mu \mathrm{m}$, and $D=40 \mu \mathrm{m}$. The input and output waveguides are configured to be operated as a single mode waveguide at the wavelength of $1.55 \mu \mathrm{m}$. To maximize the output power of $\mathrm{TE}_{0}$ mode, we define the objective function as follows:

$$
\text { Minimize } C=\left.\left.|1-| S_{21}\right|^{2}\right|^{2} \text {. }
$$

The reference index $n_{0}$ is taken to be the effective index of $\mathrm{TE}_{0}$ mode in the input waveguide. The step widths are $\Delta x=$ $0.2 \mu \mathrm{m}$ and $\Delta z=1 \mu \mathrm{m}$. The penalty parameter changes from $m=2$ to 64 linearly during the optimization process. We give an uniform refractive index distribution $(\rho=0.3)$ as an initial structure in the design region in order to show the special initial structure is not required. Unnecessary core refractive index regions appear in the optimized structure in the case that $\rho \geq 0.5$ in the initial uniform region. In addition, too small value of $\rho$ leads to a nearly zero sensitivity, thus the device performance is not efficiently improved in this optimization process.

The normalized output power as a function of the number of iterations is shown by the solid curve in Fig. 5. We can see that the transmission property is improved with the iteration. The optimized refractive index distribution of the S-bend waveguide and its field pattern are shown in Fig. 6. Offsets of the waveguide for mode matching at the input and output ports, and the grating-like structures emerge to suppress the radiation waves in the optimized distribution. The normalized output power in the binarized optimized refractive index distribution is 0.941 . Considering practical production, it is preferable that a fine structure does not emerge. The dashed curve shown in Fig. 5 is the normalized output power as a function of the number of iterations in the case of using a smoothing filter which is a simple moving average filter with $3 \times 3$ kernel size. The discretized density parameter distribution $\rho_{i, j}$ is filtered

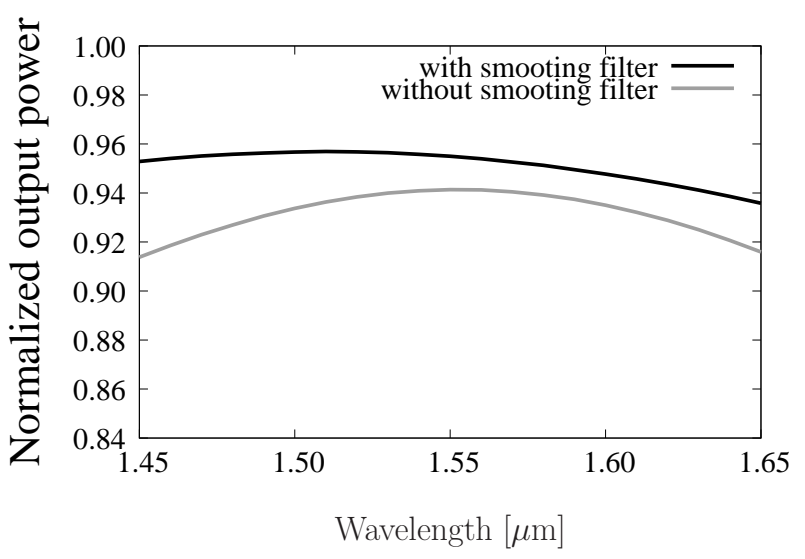

Fig. 8. Wavelength dependence of the normalized output power of the optimized S-bend waveguides.

at each iteration as follows:

$$
\rho_{i, j}^{\text {filtered }}=\sum_{\xi=-1}^{1} \sum_{\eta=-1}^{1} \frac{1}{9} \rho_{i+\xi, j+\eta} .
$$

Although the output power vibrates marginally in consequence of the smoothing filter, the transmission property in the optimized S-bend is almost the same with the previous results. The optimized refractive index distribution and its field pattern using the smoothing filter are shown in Fig. 7. Compared with Fig. 6(a), we can see that a simpler refractive index distribution is obtained. The normalized output power in the binarized optimized refractive index distribution is 0.955 when the smoothing filter is employed.

Figure 8 shows the wavelength dependence of the normalized output power of optimized S-bend waveguides. Although the grating-like structure emerges in optimized S-bend waveguide, broadband property is obtained especially in the case of using the smoothing filter. The designed structure shown in Fig. 7(a) is required to be insensitive to the slight structural deviation induced by the smoothing filter. Consequently, using smoothing filter seems to lead to the optimized structure whose transmission property is insensitive to wavelength.

\section{B. Y-branch waveguide}

In this subsection, we consider a weakly guiding 2-D Ybranch waveguide which divides the input power equally into two ports in this example. The design model of a Y-branch waveguide is shown in Fig. 9. The configuration parameters of the design model are as follows: $w=5 \mu \mathrm{m}, L=500 \mu \mathrm{m}$, $d_{1}=50 \mu \mathrm{m}, d_{2}=30 \mu \mathrm{m}, l=50 \mu \mathrm{m}$, and $D=40 \mu \mathrm{m}$. In order to equally divide the power into port 2 and 3 , we set the objective function as follows:

$$
\text { Minimize } C=\left.\left.\left|\frac{1}{2}-\right| S_{21}\right|^{2}\right|^{2}+\left.\left.\left|\frac{1}{2}-\right| S_{31}\right|^{2}\right|^{2} .
$$

In addition, we impose a symmetry condition to keep the power division equally into port 2 and 3 . The other design conditions are same as the previous design example. 


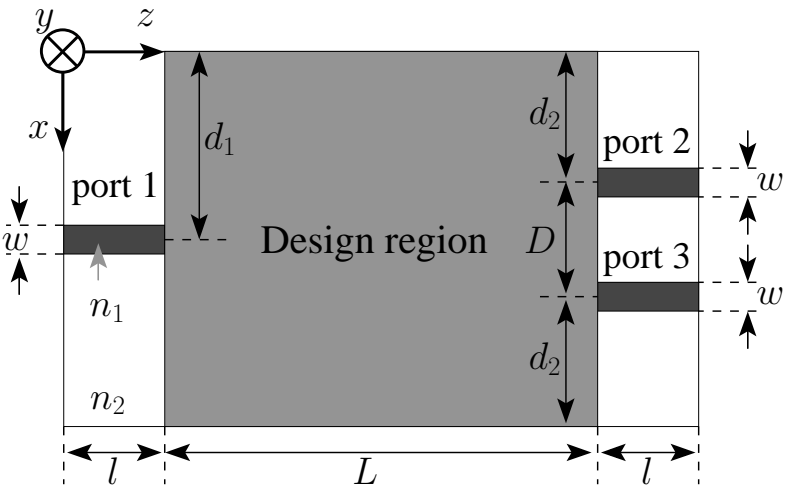

Fig. 9. A design model of a 2-D Y-branch waveguide.

The normalized output power in port 2 and 3 as a function of the number of iterations are shown in Fig. 10. In this design example also, the transmission characteristics are improved with the iteration. The normalized output power with the binarized optimized refractive index distribution is 0.490 in each port. The optimized refractive index distribution of the Y-branch waveguide and the field pattern are shown in Fig. 11. In the vicinity of the input port, the structure which can reduce the radiation into the longitudinal direction emerges. Y-branch profile similar to this structure was proposed, for instance in [22]. Moreover, as is the case in the design of the S-bend waveguide, offsets in the both output ports and the grating-like structure emerge in the optimized structure.

Our approach is based on an objective function minimization problem. Thus, for instance, $(X: Y)$ power divider can be designed by setting the objective function as follows:

$$
\begin{gathered}
\text { Minimize } C=W_{1} C_{1}+W_{2} C_{2}, \\
C_{1}=\left.|X| S_{31}\right|^{2}-\left.Y\left|S_{21}\right|^{2}\right|^{2}, \\
C_{2}=\left|1-\left(\left|S_{21}\right|^{2}+\left.\left|S_{31}\right|\right|^{2}\right)\right|^{2},
\end{gathered}
$$

where $W_{1}$ and $W_{2}$ are the weighting parameters and are taken

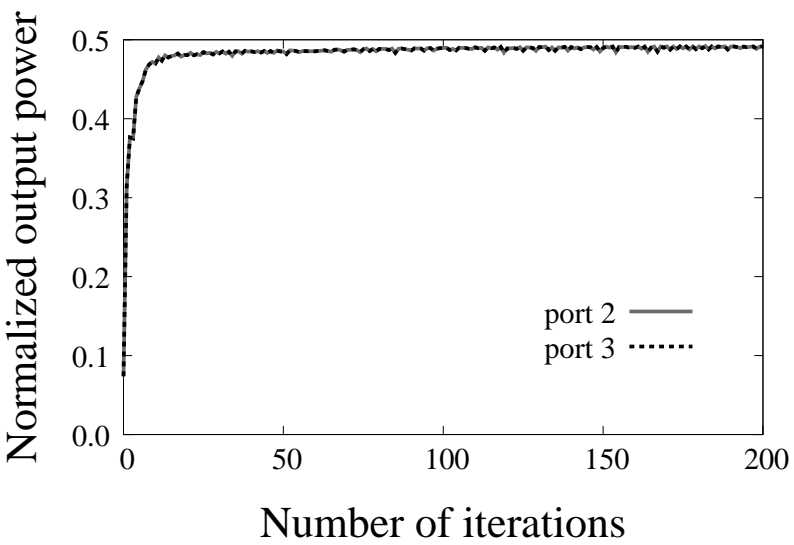

Fig. 10. The normalized output power of the Y-branch waveguide in port 2 and port 3 as a function of the number of iterations.

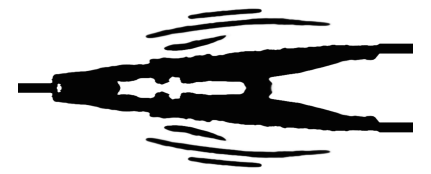

(a) Optimized refractive index distribution

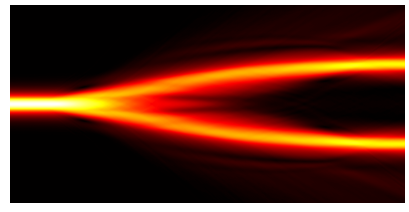

(b) Field pattern $\left(\left|E_{y}\right|\right)$
Fig. 11. The refractive index distribution and the field pattern of the optimized Y-branch waveguide using a smoothing filter.

to be,

$$
W_{1}=\frac{C_{1}}{C_{1}+C_{2}}, W_{2}=\frac{C_{2}}{C_{1}+C_{2}} .
$$

Due to the weighting parameters, larger one of them, $C_{1}$ and $\mathrm{C}_{2}$, is minimized preferentially.

Using the optimized Y-branch structure as the initial refractive index distribution shown in Fig. 11(a) and the smoothing filter, we design three power dividers which have different splitting ratios. The structure of optimized $(X: Y)$ power divider and its field patters are shown in Fig. 12. The insertion loss and the splitting ratio of each optimized structure at a wavelength of $1.55 \mu \mathrm{m}$ and the wavelength dependence are shown in Table I and Fig. 13, respectively. We can see that a low wavelength dependence is realized in the optimized power dividers owing to using the smoothing filter although larger splitting ratio causes larger insertion loss and greater wavelength dependence of the splitting ratio. As described in [9], an objective function taking account of the wavelength dependence should be used if a desired broadband property cannot be obtained. As shown in these design examples, an optical waveguide device which is hard to design based on theoretical approach can be found out automatically by using our approach.

TABLE I

THE INSERTION LOSS AND THE SPLITTING RATIO OF THE OPTIMIZED $(X: Y)$ POWER DIVIDER.

\begin{tabular}{lcc}
\hline \hline & Insertion loss [dB] & Splitting ratio \\
\hline (3:2) power divider & -14.3 & 1.50 \\
$(2: 1)$ power divider & -16.0 & 2.00 \\
(3:1) power divider & -11.9 & 2.92 \\
\hline \hline
\end{tabular}

\section{Conclusion}

We presented a new approach of topology optimization of optical waveguides using the AVM and the BPM, and described the way to evaluate the sensitivities in the case of using the density method. We are the first to apply the topology optimization utilizing the BPM and the AVM to the design of weakly guiding waveguide devices.

We demonstrated that the low loss S-bend and Y-branch are found out by using our approach, therefore the validity of our approach is confirmed. Arbitrary transmission properties are configurable in our approach thus it can be applied to a design of an arbitrary waveguide device which has an another function, such as a $\mathrm{X}$-junction, a mode/wavelength/polarization 


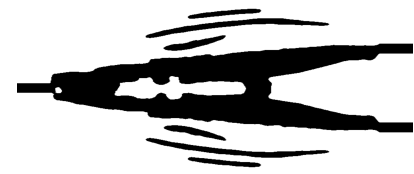
distribution

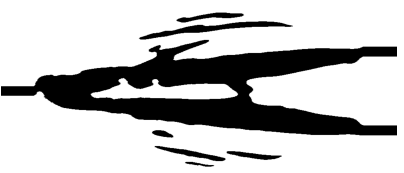

(c) Optimized refractive index distribution

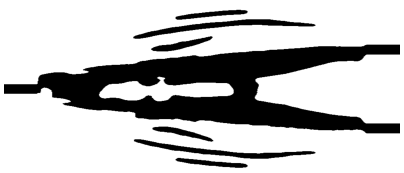

(e) Optimized refractive index distribution (a) Optimized refractive index

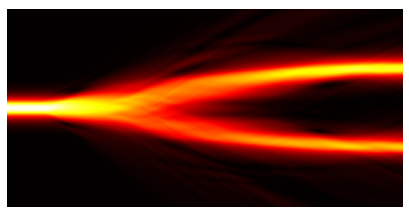

(b) Field pattern $\left(\left|E_{y}\right|\right)$

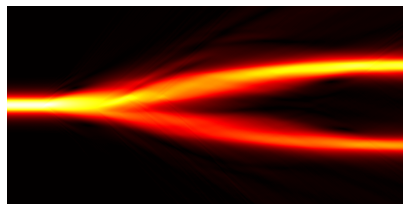

(d) Field pattern $\left(\left|E_{y}\right|\right)$

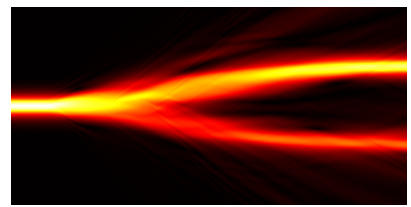

(f) Field pattern $\left(\left|E_{y}\right|\right)$
Fig. 12. The refractive index distribution and the field pattern of the optimized power divider using a smoothing filter. (a) and (b): (3:2) power divider, (c) and (d): (2:1) power divider, (e) and (f): (3:1) power divider.

splitter, and so on. It is our future work to design these kind of devices using our topology optimization approach.

In the case of utilizing topology optimization, arbitrary refractive index distribution can be appeared in the design region. The optimized devices shown in this paper are weakly guiding and these distributions do not violate the assumption of the BPM. However, in particular strongly guiding optical waveguides, the assumption may be violated. Topology optimization based on the FEM or FDTD has been successfully applied to strongly guiding waveguide problems [1]- [6], [9][11]. Thus, the applicability of our approach is principally limited by the assumption. It is known that the accuracy of the BPM is degraded at an abrupt refractive index change area. Therefore, our approach could be applied to the design of strongly guiding optical waveguides by developing some additional constraint condition to suppress an emergence of the abrupt refractive index change. It is also our future work.

\section{ACKNOWLEDGEMENT}

This work was supported by JSPS KAKENHI Grant Number $15 \mathrm{~K} 06009$.

\section{REFERENCES}

[1] J. S. Jensen and O. Sigmund, "Systematic design of photonic crystal structures using topology optimization: Low-loss waveguide bends," Appl. Phys. Lett., Vol. 84, No. 12, pp. 2022-2024, Mar. 2004.

[2] J. S. Jensen, O. Sigmund, L. H. Frandsen, P. I. Borel, A. Harpth, and M. Kristensen, "Topology design and fabrication of an efficient double 90 photonic crystal waveguide bend," IEEE Photon. Technol. Lett., Vol. 17, No. 6, pp. 1202-1204, June 2005.

[3] P. I. Borel, A. Harpth, L. H. Frandsen, M. Kristensen, P. Shi, J. S. Jensen, and O. Sigmund, "Topology optimization and fabrication of photonic crystal structures," Opt. Express, Vol. 12, No. 9, pp. 1996-2001, May. 2004.

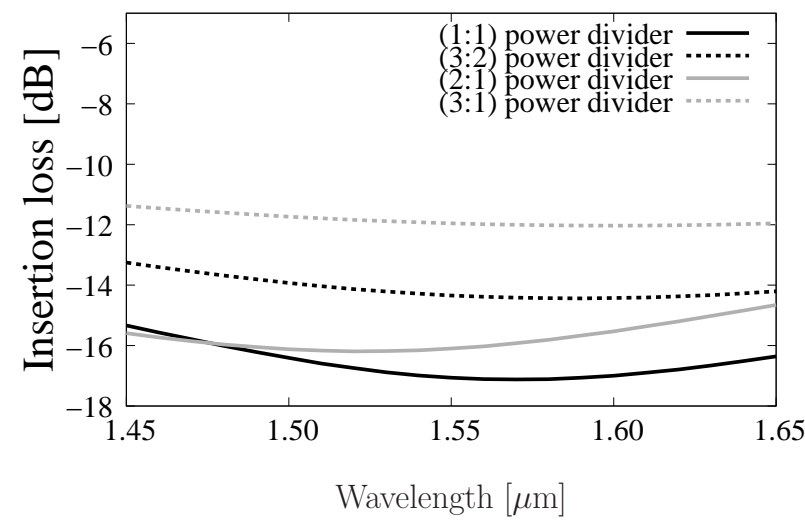

(a) Insertion loss

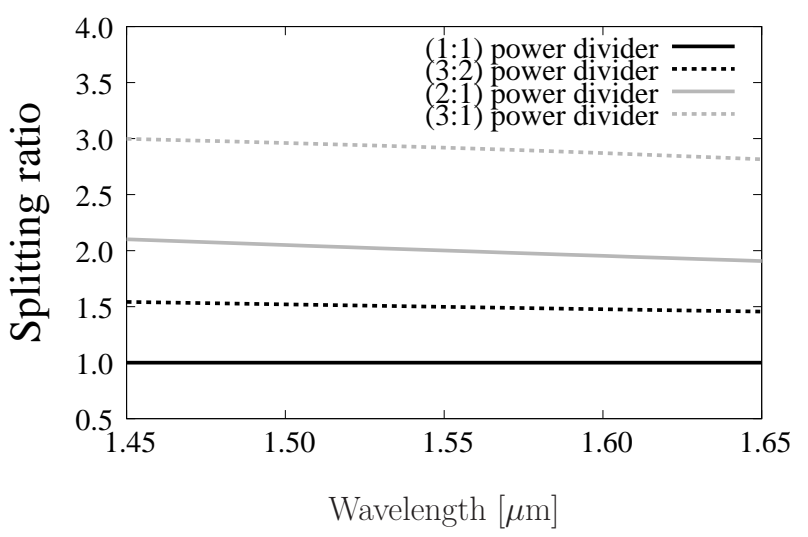

(b) Splitting ratio

Fig. 13. Wavelength dependence of the optimized power dividers.

[4] P.I. Borel, L.H. Frandsen, A. Harpth, M. Kristensen, J. S. Jensen, and O. Sigmund, "Topology optimised broadband photonic crystal Y-splitter," Electron. Lett., Vol. 41, No. 2, pp. 69-71, Jan. 2005.

[5] R. Matzen, J. S. Jensen, and O. Sigmund, "Systematic design of slowlight photonic waveguides," J. Opt. Soc. Amer. B, Vol. 28, No. 10, pp. 2374-2382, Oct. 2011.

[6] L. H. Frandsen, Y. Elesin, L. F. Frellsen, M. Mitrovic, Y. Ding, O. Sigmund, and K. Yvind, "Topology optimized mode conversion in a photonic crystal waveguide fabricated in silicon-on-insulator material," Opt. Express, Vol. 22, No. 7, pp. 8525-8532, Apr. 2014.

[7] Y. Tsuji, K. Hirayama, T. Nomura, K. Sato, S. Nishiwaki, "Design of optical circuit devices based on topology optimization," IEEE Photon. Technol. Lett., Vol. 18, No. 7, pp. 850-852, Apr. 2006

[8] Y. Tsuji and K. Hirayama, "Design of optical circuit devices using topology optimization method with function-expansion-based refractive index distribution," IEEE Photon. Technol. Lett., Vol. 20, No. 12, pp. 982-984, June 2008

[9] K. Fujimoto, Y. Tsuji, K. Hirayama, T. Yasui, S. Sato, and R. Kijima, "A study on topology optimization of optical circuits consisting of multimaterials," J. Lightw. Technol., Vol. 30, No. 13, pp. 2210-2215, July 2012.

[10] T. Yasui, Y. Tsuji, J. Sugisaka, and K. Hirayama, "Design of threedimensional optical circuit devices by using topology optimization method with function-expansion-based refractive index distribution," $J$. Lightw. Technol., Vol. 31, No. 23, pp. 3765-3770, Dec. 2013.

[11] Z. Zhang, Y. Tsuji, T. Yasui, and K. Hirayama, "Design of ultra-compact triplexer with function-expansion based topology optimization," Opt. Express, Vol. 23, No. 4, pp. 3937-3950, Feb. 2015.

[12] T. Hashimoto, T. Saida, I. Ogawa, M. Kohtoku, T. Shibata, and H. Takahashi, "Optical circuit design based on a wavefront-matching method," Opt. Lett., Vol. 30, No. 19, pp. 2620-2622, Oct. 2005.

[13] Y. Sakamaki, T. Saida, T. Hashimoto, and H. Takahashi, "New optical 
waveguide design based on wavefront matching method," J. Lightw. Technol., Vol. 25, No. 11, pp. 3511-3518, Nov. 2007.

[14] Y. Sakamaki, T. Saida, T. Shibata, Y. Hida, T. Hashimoto, M. Tamura, and H. Takahashi, "Y-branch waveguides with stabilized splitting ratio designed by wavefront matching method," IEEE Photon. Technol. Lett., Vol. 18, No. 7, pp. 817-819, Apr. 2006.

[15] Y. Sakamaki, T. Saida, T. Hashimoto, S. Kamei, and H. Takahashi, "Loss reduction of waveguide crossings by wavefront matching method and their application to integrated optical circuits," J. Lightw. Technol., Vol. 27, No. 13, pp. 2257-2263, July 2009.

[16] Y. Sakamaki, S. Kamei, T. Hashimoto, T. Kitoh, and H. Takahashi, "Loss uniformity improvement of arrayed-waveguide grating with mode-field converters designed by wavefront matching method," J. Lightw. Technol., Vol. 27, No. 24, pp. 5170-5715, Dec. 2009.

[17] M. A. Swillam, M. H. Bakr, and X. Li, "Efficient adjoint sensitivity analysis exploiting the FD-BPM," J. Ligthw. Technol., Vol. 25, No. 7, pp. 1861-1869, July 2007.

[18] M. A. Swillam, M. H. Bakr, and X. Li, "Full vectorial 3-D sensitivity analysis and design optimization using BPM," J. Lightw. Technol., Vol. 26, No. 5, pp. 528-536, Mar. 2008.

[19] Y. Chung and N. Dagli, "An assessment of finite difference beam propagation method," IEEE J. Quantum Electron., Vol. 26, No. 8, pp. 1335-1339, Aug. 1990.

[20] M. S. Stern, "Semivectorial polarised H field solutions for dielectric waveguides with arbitrary index profiles," IEE Proc. J, Vol. 135, No. 5, pp. 333-338, Oct. 1988.

[21] G. R. Hadley, "Transparent boundary condition for beam propagation," Opt. Lett., Vol.16, No. 9, pp. 624-626, May. 1991.

[22] Z. Weissman, E. Marom, and A. Hardy, "Very low-loss Y-junction power divider," Opt. Lett., Vol.14, No. 5, pp. 293-295, Mar. 1989.

Akito Iguchi received the B.S. degree in information and electronic engineering from Muroran Institute of Technology, Muroran, Japan, in 2015. He is presently studying toward the M.S. degree in information and electronic engineering at Muroran Institute of Technology.

Mr. Iguchi is a student member of the Institute of Electronics, Information and Communication Engineers (IEICE) and IEEE.

Yasuhide Tsuji (M'97) received the B.S., M.S., and Ph.D. degrees in electronic engineering from Hokkaido University, Sapporo, Japan, in 1991, 1993, and 1996, respectively.

In 1996, he joined the Department of Applied Electronic Engineering, Hokkaido Institute of Technology, Sapporo, Japan. From 1997 to 2004, he was an Associate Professor of Electronics and Information Engineering at Hokkaido University. From 2004 to 2011, he was an Associate Professor of Electrical and Electronic Engineering at Kitami Institute of Technology, Kitami, Japan. Since 2011, he has been a Professor of Information and Electronic Engineering at Muroran Institute of Technology, Muroran, Japan. He has been interested in wave electronics.

Dr. Tsuji is a member of the Institute of Electronics, Information and Communication Engineers (IEICE), the Japan Society of Applied Physics, the Optical Society of America (OSA), and IEEE. In 1997 and 1999, he was awarded the Excellent Paper Award from IEICE. In 2000, he has received the Third Millennium Medal from IEEE.

Koichi Hirayama (SM'96) received the B.S., M.S., and Ph.D. degrees in electronic engineering from Hokkaido University, Sapporo, Japan, in 1984, 1986, and 1989, respectively.

In 1989, he joined the Department of Electronic Engineering, Kushiro National College of Technology, Kushiro, Japan. In 1992, he became an Associate Professor of Electronic Engineering at Kitami Institute of Technology, Kitami, Japan, and in 2004 he became a Professor. He has been interested in the analysis and optimal design of electromagnetic and optical waveguides.

Dr. Hirayama is a member of the Institute of of Electronics, Information and Communication Engineers (IEICE) of Japan and the Japan Society of Applied Physics.
Takashi Yasui (S'00-M'02) received the B.S. degree in electronic engineering from Fukui University, Fukui, Japan, in 1997, and the M.S. and Ph.D. degrees in electronic engineering from Hokkaido University, Sapporo, Japan, in 1999 and 2001, respectively.

From 1999 to 2002, he was a Research Fellow of the Japan Society for the Promotion of Science. In 2002, he joined Fujitsu Ltd., Chiba, Japan. From 2004 to 2011, he was an Assistant Professor of the Department of Electronic and Control Systems Engineering, Shimane University, Matsue, Japan. Since 2011, he has been an Associate Professor of the Department of Electrical and Electronic Engineering, Kitami Institute of Technology, Kitami, Japan. He has been engaged in research on wave electronics.

Dr. Yasui is a member of the Applied Computational Electromagnetics Society, the Optical Society of America, the Institute of Electronics, Information, and Communication Engineers of Japan, and the Magnetics Society of Japan. 